\title{
Gingival Biotype in the Maxillary Esthetic Zone: A Pilot Study comparing Three Noninvasive Assessment Methods
}

\author{
${ }^{1}$ Sunil K Nettemu, ${ }^{2}$ Sowmya Nettem, ${ }^{3}$ Bryan J Paulose, ${ }^{4}$ Vi V How, ${ }^{5}$ Vijendra P Singh
}

\begin{abstract}
This pilot study aims to compare reliability of visual, probe transparency, and soft tissue cone beam computed tomography (CBCT) methods for estimating gingival biotype in maxillary esthetic zone. This study included 10 patients with indications for dental implant therapy in the esthetic zone of maxilla. Visually, the gingival biotype in the maxillary esthetic zone was examined by two examiners. Subsequently, the gingival sulcus was probed and observed for its transparency, and soft tissue thickness was measured using soft tissue CBCT. A 100\% correlation was found between probe transparency and visual inspection. When comparing visual inspection and probe transparency to soft tissue CBCT, a statistical discrepancy of $57.1 \%$ was found. However, the $p$-value of 0.125 indicated a nonsignificant difference. As per our knowledge, this is the first study comparing the reliabilities of these three noninvasive biotype assessments.
\end{abstract}

Keywords: Biotype, Maxillary esthetic zone, Osseointegrated implants, Probe transparency.

How to cite this article: Nettemu SK, Nettem S, Paulose BJ, How VV, Singh VP. Gingival Biotype in the Maxillary Esthetic Zone: A Pilot Study comparing Three Noninvasive Assessment Methods. Int J Prosthodont Restor Dent 2016;6(4):85-88.

\section{Source of support: Nil}

Conflict of interest: None

\section{INTRODUCTION}

Complete harmony and symmetry of a restoration with the surrounding soft tissues may be the most challenging, yet the ultimate goal of therapy in terms of esthetics. An insight into the gingival biotype is a prerequisite to accomplish this goal. The identification of the gingival biotype is

\footnotetext{
${ }^{1}$ Associate Professor and Head, ${ }^{2,5}$ Associate Professor ${ }^{3,4}$ Dental Officer

1,2,5Department of Periodontics, Faculty of Dentistry, MelakaManipal Medical College, Melaka, Malaysia

${ }^{3}$ Department of General Dentistry, Ministry of Health, Division of Oral Health, Miri, Sarawak, Malaysia

${ }^{4}$ Department of General Dentistry, Ministry of Health, Division of Oral Health, Jelebu, Negeri Sembilan, Malaysia
}

Corresponding Author: Sowmya Nettem, Associate Professor Department of Periodontics, Faculty of Dentistry, Melaka-Manipal Medical College, Melaka, Malaysia, Phone: +0060105177660 e-mail: drsowmyark@gmail.com the most important and most often overlooked parameter in clinical practice. Gingival biotype is one of the key elements for deciding a successful treatment outcome in implant restorations. ${ }^{1,2}$ The clinical appearance of healthy periodontal tissues differs from subject to subject. The bulky, slightly scalloped marginal gingiva with thick underlying bony architecture and short and wide teeth was termed as "thick biotype," and the thin, highly scalloped marginal gingiva with thin underlying bone and slender tooth form was termed as "thin biotype." 3 The differences in gingival and osseous architecture have been shown to exhibit a significant impact on the outcome of periodontal, restorative, and implant therapy. ${ }^{4}$ Also, the thickness of the soft tissues can negatively influence the outcome of regenerative surgery. ${ }^{5,6}$ The observations from all these studies illustrate that disparities in esthetic treatment outcome could arise as a result of variability in tissue response to therapeutic procedures. The use of simple and reliable methods to identify gingival biotype would benefit to modulate the treatment for the individual and predict its specific outcome. ${ }^{4}$

The main problem for clinicians in immediate implant placement is the unpredictability of long-term soft tissue stability. ${ }^{7}$ The prevalence of implant-based reconstruction in the esthetic zone has risen and as a prerequisite for a successful treatment, adequate soft tissue quantity and quality are required. Therefore, the impact of treatment to be performed in the esthetic zone should not be taken lightly.

To date, with best of our knowledge, no study has been conducted to compare the reliability of different noninvasive biotype assessment methods of visual examination, probe transparency assessment, and soft tissue cone beam computed tomography (ST-CBCT). Hence, this study was undertaken to determine the most reliable method for gingival biotype assessment among three noninvasive methods.

\section{MATERIALS AND METHODS}

\section{Study Design}

In this study, the thickness of the maxillary anterior mucosa was evaluated in 10 patients ( 4 men, 6 women) with age range of 25 to 50 years. The patients were selected based on the oral implications for CBCT and dental implant 


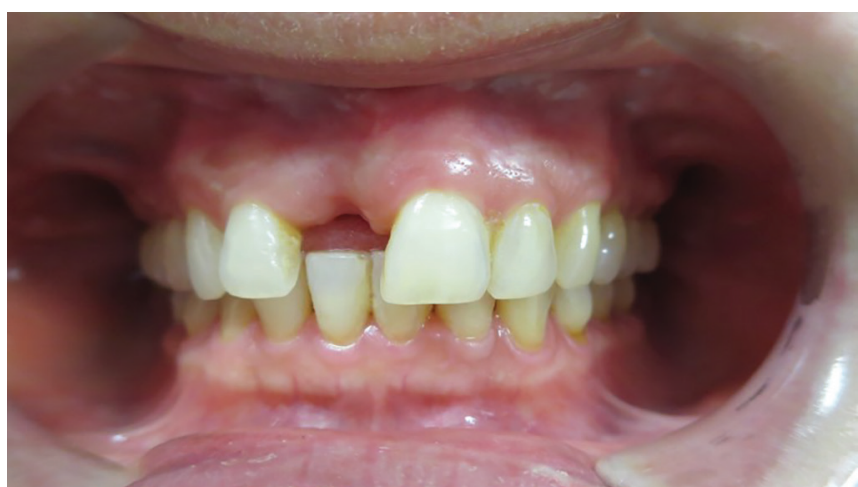

Fig. 1: Visual inspection method wherein the operator visually evaluates the gingival biotype for the anterior maxillary zone extending from canine to canine

placement at the Department of Oral Implantology, Faculty of Dentistry, Melaka-Manipal Medical College, Malaysia. The study was conducted in accordance with and approval from the Research and Ethics Committee, Faculty of Dentistry, Melaka-Manipal Medical College, Malaysia. Written informed consent was obtained from all volunteering patients. Patients eligible for this study according to the inclusion criteria included those with clinically healthy gingiva, indicated for dental implant placement in the maxillary anterior region, and presence of a minimum of one central incisor. Exclusion criteria for the study were clinical evidence of gingival or periodontal diseases, pregnant and lactating women, and patients under medications, which can induce gingival enlargement. No external funding was received for this study.

In the present study, an examination and diagnosis form includes patient's complaint, history, gingival and periodontal status, and gingival biotype assessment. The inclusion of complaint in the form shows that these patients are representing purposive sampling and no unnecessary stress and radiation exposure is subjected to the patients. History in the form is mainly to avoid patients with any medical or drug history that can influence the gingival thickness.

\section{Clinical Assessment}

The thickness of gingiva on the maxillary central incisor adjacent to the missing tooth was evaluated and categorized by two examiners. The evaluation of the gingival biotype was based on visual examination and probe transparency method. ${ }^{8}$ Patient's cheeks were retracted with a cheek retractor, so that better visualization could be achieved as shown in Figure 1, and based on visually checking the thickness of gingival biotype, it was categorized into either of the two biotypes (thick or thin). William's graduated periodontal probe was then inserted into the free gingival margin up to the base of the pocket as shown in Figure 2. If the outline of the underlying

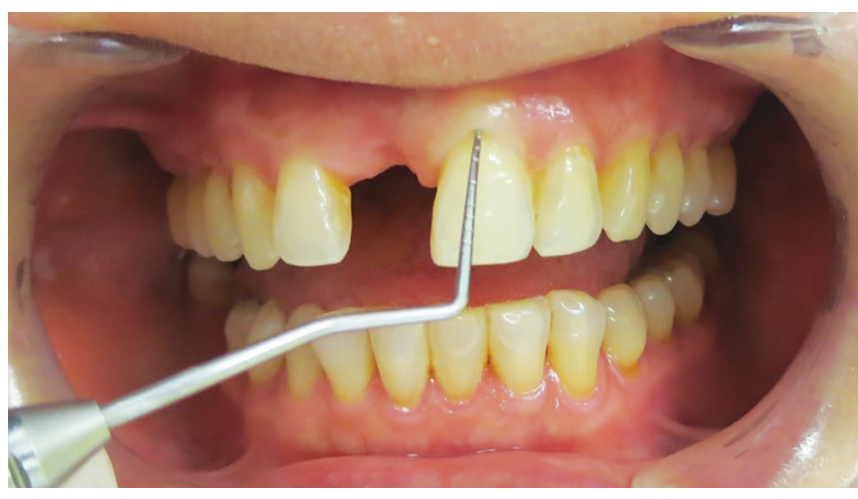

Fig. 2: Probe transparency method wherein the operator insert the periodontal probe into gingival sulcus of maxillary anterior teeth from canine to canine to check probe visibility and, thus, evaluate gingival biotype

periodontal probe ${ }^{8}$ could be visualized through the gingival tissues, it was categorized as thin biotype; if the outline of the underlying periodontal probe could not be visualized through the gingival tissues, it was categorized as thick biotype.

\section{Radiological Assessment}

The ST-CBCT scans were done using Planmeca 3Ds CBCT machine Romexis 3.0.1R with a standard investigation protocol of maximum $90 \mathrm{kVp}$, maximum $12 \mathrm{~mA}$, and field of view $(F O V)=5 \times 8 \mathrm{~cm}$. Patients' chin and heads were stabilized for the ST-CBCT scanning. The variant CBCT procedure carried out in this study was in accordance with the procedure explained by Januário et al. ${ }^{9}$ Cheek retractor was placed in an inverted position to avoid hitting on the chin stabilizer as shown in Figure 3. This procedure was done so that the soft tissues of the lips were positioned away from the gingival tissue. The images were generated and saved in the patient's folders until analysis. Figure 4 shows the measurements performed with the Romexis Measurement tool software (3.0.1R). Voxel size of $200 \mu \mathrm{m}$ was used. The contrast and brightness of the image were

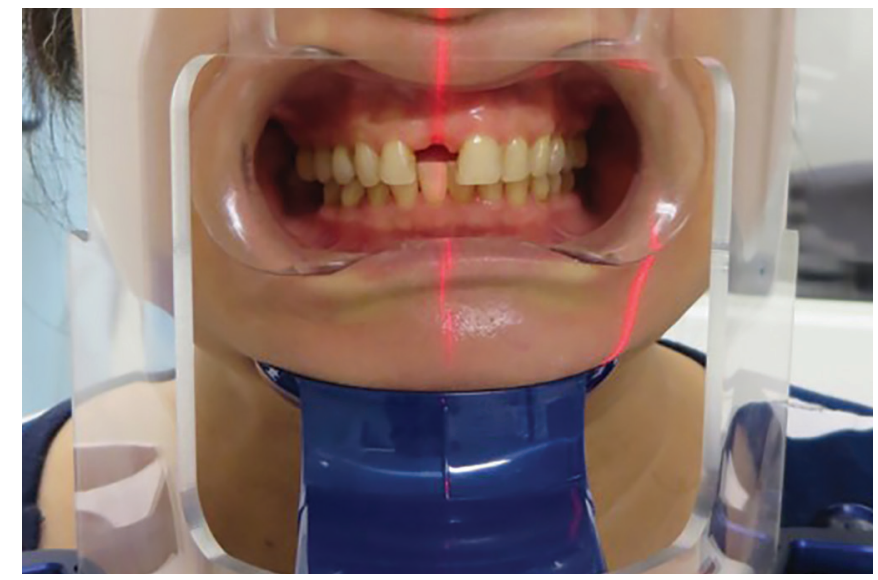

Fig. 3: Soft tissue cone beam computed tomography method wherein the cheek retractor is placed in inverted position to retract the lip while obtaining CBCT image of gingival tissue in maxillary anterior zone 


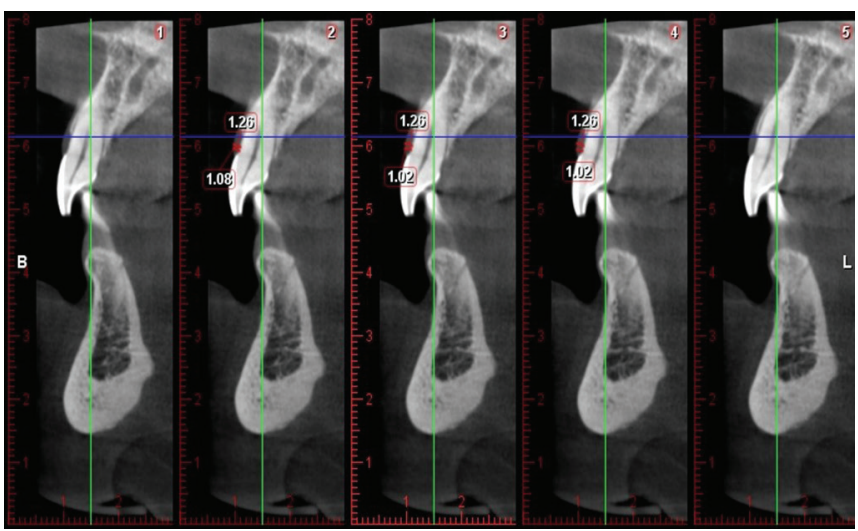

Fig. 4: Cone beam computed tomography readings of biotype thickness

adjusted, and image was magnified to appreciate the soft tissue. A perpendicular line was drawn from the labial gingival surface to the tooth surface. Two measurements were taken, one apical to marginal gingiva and one at the center of visible gingiva. An average of both the measurements was calculated. The gingival biotype was considered thin if the measurement was $<1.5 \mathrm{~mm}$ and thick if measured $\geq 1.5 \mathrm{~mm}$. $^{10}$

\section{Statistical Analysis}

The data analysis was performed using Statistical Package for the Social Sciences version 21 (IBM SPSS Statistics for Windows, Version 21.0; IBM Corp., Armonk, NY, USA). McNemar test was used to compare the data between visual examination, probe transparency, and ST-CBCT methods. Binomial distribution was used and $\mathrm{p}<0.05$ was considered statistically significant. The analysis showed no statistically significant difference between the identification of gingival biotypes using the visual inspection and probe transparency methods. Similarly, all the three noninvasive assessment methods (visual inspection, probe transparency, and ST-CBCT) showed similar identification of gingival biotypes as thick or thin and had no significant differences.

\section{RESULTS}

Based on the analysis of results, there is no significant difference of proportion of thick and thin classified in visual inspection and probe. All gingival biotypes classified as thin in visual inspection are also classified as thin in probe method (100\% correlation). Also, there is no significant difference of proportion of thick and thin classified in visual inspection and ST-CBCT. About $57.1 \%$ of thick classified in visual are classified as thin in ST-CBCT.

\section{DISCUSSION}

Gingival biotype is one of the important key elements in determining the success of an implant treatment as it provides the esthetic point of the restorative therapy. Patients with thick gingiva have been shown to have more esthetically pleasing results compared with patients with thin gingival. ${ }^{6,11-13}$ Studies have shown, in fact, that periimplant tissues, in general, are much thicker than periodontal tissues and by placing the implant palatal to the original tooth root position, we may actually be shifting the patient's biotype from thin to thick, for that particular site. ${ }^{14}$ However, no well-defined method to actually sort and classify the various human gingival biotypes is available. ${ }^{14}$ In this report, we compare three noninvasive assessment methods in obtaining the gingival biotype by using visual inspection, probe transparency method, and ST-CBCT scan. These procedures were only performed once to avoid high radiation dose and reduce the additional stress for the patients. ${ }^{7}$

Gingival and periodontal status is one of the key elements in determining the esthetics of the therapy, as it provides the width and thickness of the gingiva, degree of keratinization, color, and papilla height. ${ }^{7}$ If the gingiva is thin, but the color and degree of keratinization are high, it can increase the esthetic outcome of the implant treatment. According to the results of our study, there is no significant difference of proportion of thick and thin classified in visual inspection and probe transparency methods, whereas $57.1 \%$ of thick gingival biotypes classified using visual inspection and probe transparency methods were classified thin with the ST-CBCT method. Hence, statistically, visual inspection and probe transparency methods prove to be sufficient for assessment of gingival biotype; nevertheless, ST-CBCT scan remains a quantitative method. The results of this study are in agreement to the findings from another study, which states that CBCT measurements were an accurate representation of the clinical thickness of both labial gingiva and bone. ${ }^{15}$ Also, the thickness of the labial gingiva had a moderate association with the underlying bone radiographically. ${ }^{15}$ Hence, statistically, visual inspection and probe transparency methods prove to be sufficient for assessment of gingival biotype; nevertheless, ST-CBCT scan remains a quantitative method and, hence, has the ability of reproducing the same results at different time frames. Visual inspection is highly dependent on examiner's subjective observation; hence, it is subject to interexaminer variability. Also, simple visual inspection may not be considered a valuable method as nearly half of the high-risk patients with thin scalloped biotype were overlooked. ${ }^{16}$ Clinically, ST-CBCT scan produces more objective results as compared with the other two assessment methods as it produces a quantitative result.

The results in this pilot study are based on a small sample size of patients, which could be the reason for the large difference in percentage; future research is required to be carried out in this direction. 


\section{CONCLUSION}

Statistically, there is no significant difference of p-value between visual and ST-CBCT. However, clinically, a discrepancy of $57.1 \%$ between thick and thin gingiva findings is of significant concern. Further studies with a larger sample size will definitely prove beneficial in achieving superior esthetic and functional outcomes in the practice of implant dentistry.

\section{REFERENCES}

1. Kois JC. Predictable single-tooth peri-implant esthetics: five diagnostic keys. Compend Contin Educ Dent 2004 Nov;25(11):895-896, 898, 900.

2. Kao RT, Fagan MC, Conte GJ. Thick vs. thin gingival biotypes: a key determinant in treatment planning for dental implants. J Calif Dent Assoc 2008 Mar;36(3):193-198.

3. Olsson M, Lindhe J. Periodontal characteristics in individuals with varying form of the upper central incisors. J Clin Periodontol 1991 Jan;18(1):78-82.

4. De Rouck T, Eghbali R, Collys K, De Bruyn H, Cosyn J. The gingival biotype revisited: transparency of the periodontal probe through the gingival margin as a method to discriminate thin from thick gingiva. J Clin Periodontol 2009 May;36(5): 428-433.

5. Anderegg CR, Metzler DG, Nicoll BK. Gingival thickness in guided tissue regeneration and associated recession at facial furcation defects. J Periodontol 1995 May;66(5):397-402.

6. Baldi C, Pini-Prato G, Pagliaro U, Nieri M, Saletta D, Muzzi L, Cortellini P. Coronally advanced flap procedure for root coverage. Is flap thickness a relevant predictor to achieve root coverage? A 19-case series. J Periodontol 1999 Sep;70(9): 1077-1084.
7. Dvorak G, Arnhart C, Schön P, Heuberer S, Watzek G, Gahleitner A. The "puffed cheek method" to evaluate mucosal thickness: case series. Clin Oral Implants Res 2013 Jul;24(7):719-724.

8. Kan JY, Morimoto T, Rungcharassaeng K, Roe P, Smith DH. Gingival biotype assessment in the esthetic zone: visual versus direct measurement. Int J Periodontics Restorative Dent 2010 Jun;30(3):237-243.

9. Januário AL, Barriviera M, Duarte WR. Soft tissue cone-beam computed tomography: a novel method for the measurement of gingival tissue and the dimensions of the dentogingival unit. J Esthet Restor Dent 2008 Dec;20(6):366-374.

10. Greenberg J, Laster L, Listgarten MA. Transgingival probing as a potential estimator of alveolar bone level. J Periodontol 1976 Sep;47(9):514-517.

11. Huang LH, Neiva RE, Wang HL. Factors affecting the outcomes of coronally advanced flap root coverage procedure. J Periodontol 2005 Oct;76(10):1729-1734.

12. Hwang D, Wang HL. Flap thickness as a predictor of root coverage: a systematic review. J Periodontol 2006 Oct;77(10): 1625-1634.

13. Kan JY, Rungcharassaeng K, Umezu K, Kois JC. Dimensions of peri-implant mucosa: an evaluation of maxillary anterior single implants in humans. J Periodontol 2003 Apr;74(4): 557-562.

14. Cabello G, Rioboo M, Fábrega JG. Immediate placement and restoration of implants in the aesthetic zone with a trimodal approach: soft tissue alterations and its relation to gingival biotype. Clin Oral Implants Res 2013 Oct;24(10):1094-1100.

15. Fu JH, Yeh CY, Chan HL, Tatarakis N, Leong DJ, Wang HL. Tissue biotype and its relation to the underlying bone morphology. J Periodontol 2010 Apr;81(4):569-574.

16. Eghbali A, De Rouck T, De Bruyn H, Cosyn J. The gingival biotype assessed by experienced and inexperienced clinicians. J Clin Periodontol 2009 Nov;36(11):958-963. 